\title{
Medios de comunicación como agentes que facilitan la medicalización de la vida: el ejemplo de la andropausia
}

\author{
Enrique Gavilán. \\ Servicio Extremeño de Salud \\ (Cáceres, España) \\ Ainhoa Iriberri. \\ Periodista freelance especialista en biomedicina. \\ (Madrid, España)
}

Palabras clav
Periodismo
medicalización
andropausia
madurez y
envejecimiento
sexualidad
mercadotecnia

\begin{abstract}
Resumen
A través del fenómeno de la medicalización, multitud de procesos que anteriormente eran considerados ajenos a la jurisdicción de la medicina han pasado a ser explicados y tratados por médicos. Uno de ellos es el proceso natural del envejecimiento, y dentro de él, el declive en la actividad -principalmente sexual- del varón. Clásicamente llamado andropausia y considerado como un síndrome por falta de testosterona, ha pasado por momentos históricos en los que casi parecía desaparecido del discurso médico. Sin embargo, con el auge de los medicamentos para el sexo, la idea de la andropausia ha resurgido, a pesar de que numerosas evidencias científicas acumuladas en los últimos años hayan alimentado muchas dudas alrededor de este concepto.
\end{abstract}

La información sobre salud ayuda a normalizar los procesos sociales por los que determinados problemas caen en el dominio de la medicina. La andropausia no ha sido ajena a este fenómeno. A través del análisis de noticias aparecidas en medios españoles de prensa escrita sobre la andropausia y la sexualidad del varón maduro, en el artículo se describen algunos ejemplos que muestran cómo los medios de comunicación han podido influir en la medicalización de este proceso. Igualmente, se analizará la influencia que ejercen los grupos de presión, a través de la financiación de los grupos mediáticos, y de los conflictos de interés de medios y profesionales de la comunicación en la selección de las fuentes de información y en los contenidos de la misma, así como el papel de las agencias de prensa y las asociaciones de periodistas.

Los autores declaran no tener conflictos de interés. Enrique Gavilán forma parte del equipo editorial de Revista de Comunicación y Salud y no ha participado en el proceso de edición de este artículo. 


\title{
Media as agents that facilitate the medicalization of life: the example of andropause
}

\begin{tabular}{c}
\hline Keywords \\
\hline Journalism \\
medicalization \\
andropause \\
aging \\
sexuality \\
marketing
\end{tabular}

\begin{abstract}
Trough the phenomenon of medicalization, many processes previously beyond the jurisdiction of medicine has come to be explained and treated by doctors. One of them is the natural process of aging, and within it the decline in male activity -principally sexual. Traditionally known as andropause and considered as a syndrome caused by a lack of testosterone, there have been moments in history when it seemed to have almost disappeared from medical debate. However, with the boom of sexual drugs, the iede of andropause has reappeared, despite the accumulated scientific evidence which has fed doubt about this concept within the past 15 years.

Information about health helps to normalize social processes which have led certain problems to be considered as part of the medicine field. Andropause has not been immune to this phenomenon. Through the analysis of news articles published in the Spanish press about andropause and the sexuality of the aging male, some examples are described in this essay to show how the media can influence in the medicalization of this process. In this regard the influence of lobbies will be analyzed, through the funding of media groups and as a result of conflicts of interest among media and communication professionals when selecting information sources and content, as well as the role of news agencies and press associations.
\end{abstract}

\section{Cómo citar el artículo}

Gavilán, E.; e Iriberri, A. (2014). Medios de comunicación como agentes que facilitan la medicalización de la vida: el ejemplo de la andropausia. Revista de Comunicación y Salud, Vol. 4, pp. 49-67.

DOI: http://doi.org/10.35669/revistadecomunicacionysalud.2014.4.49-67 


\section{La medicalización del envejecimiento}

\subsection{Qué entendemos por medicalización y de dónde surge}

La medicalización es el proceso por el cual un problema cae en la jurisdicción de la medicina, siendo "definido en términos médicos, descrito usando lenguaje médico, entendido bajo la adopción de un marco médico o tratado con una intervención médica" (Conrad, 2007). Son muchos los ejemplos de procesos han sido medicalizados: conductas consideradas "desviadas" (homosexualidad, alcoholismo), problemas de adaptación (fracaso en las relaciones de pareja o el ámbito laboral), sentimientos (infelicidad) y estados (soledad, pobreza, desempleo) (Conrad 2007). Especialmente proclives son determinados momentos de vulnerabilidad, como el embarazo, la niñez, la menopausia o el envejecimiento.

Al medicalizar confiamos en que la intervención médica genere salud y bienestar, pero en ocasiones ocurre más bien lo contrario (Illich, 1975). La iatrogenia es una de las principales causas de muerte y enfermedad en EEUU (Null, 2010). Pero además, las intervenciones sanitarias no están exentas de inconvenientes (acudir a consultas, someterse a pruebas, el estigma social parejo a la etiqueta diagnóstica, insatisfacción por no cumplir las expectativas) y contribuye a disparar el gasto (Conrad, 2010).

Por todos estos motivos, el análisis de la expansión de los límites de la medicina se ha detenido más en sus consecuencias nocivas, aún cuando a veces conlleva efectos positivos (Parens, 2013). Sin duda, el desarrollo de la medicina ha contribuido a que disfrutemos de una vida más larga y de mejor calidad, aunque con una distribución muy desigual en los esfuerzos y en los resultados (Watt, 2002). En lo personal, definir un problema en términos médicos puede adquirir un valor adaptativo, al legitimar y descargar de culpa o vergüenza, a la vez que sirve de justificación de demanda de apoyo y solidaridad que muchos pacientes no encuentran en otros ámbitos (Broom y Woodward, 1996).

El afán de control y poder de la medicina es uno de los factores determinantes en el nacimiento del fenómeno de la medicalización (Conrad, 1992; Illich 1975), pero no es el único. La expansión de las economías de mercado, el crecimiento económico sostenido y la creciente influencia de la industria farmacéutica han sido fundamentales en su auge en los últimos años del siglo XX (Conrad 2007). La expansión de la cultura de consumo y los movimientos sociales y organizaciones de pacientes comienzan a jugar un papel hegemónico (Greenhalgh y Wessely, 2004; Ballard y Elston, 2005; Conrad y Leiter, 2004). Otros factores, por el contrario, contribuyen a la desmedicalización, como los límites legales y las políticas de contención de costes, la competencia entre entidades nosológicas y productos sanitarios, la propia debilidad de las evidencias y la falta de consenso en la comunidad científica (Conrad 2007; Barsky y Forus, 1995).

\subsection{La medicalización de la madurez sexual del varón}

La búsqueda del elixir de la eterna juventud es uno de los más antiguos anhelos de la humanidad. En el siglo XIX se vinculó el envejecimiento con la caída en las hormonas 
sexuales (estrógenos y testosterona). La posibilidad de enriquecerlas a partir de extractos animales, a los que se atribuían múltiples propiedades, abriría la puerta a un nuevo tipo de remedios contra el envejecimiento, que a comienzos del siglo $X X$ se hicieron muy populares (Brown-Séquard, 1889; Khan, 2005).

La testosterona finalmente se pudo sintetizar de forma artificial en 1934. La posibilidad de producirla de forma industrial facilitó el desarrollo del concepto de andropausia a partir de ese momento (Marañón, 1937). Sin embargo, desde la década de los 40 y hasta mediados de los 90 , los síntomas atribuidos a la falta de testosterona pasaron a ser explicados mejor desde la óptica de la psicología (Watkins, 2008).

Los fármacos del sexo (sildenafilo y similares) revolucionaron entonces el mercado farmacéutico, lo que permitió rescatar la idea de la andropausia como declive hormonal, redefinida ahora como una enfermedad para la cual se han barajado varios nombres (síndrome por déficit de testosterona -SDT-, hipogonadismo tardío y déficit androgénico del varón maduro). A ello contribuye la disponibilidad de nuevas presentaciones tópicas que permiten equiparar la testosterona a un bien de consumo diario (Groopman, 2002). En la promoción de esta "nueva enfermedad" se han empleado estrategias que han involucrado a medios de comunicación, expertos, asociaciones profesionales y personajes famosos (Gérvas, 2012).

Sin embargo, la comunidad científica se resiste a aceptar que el síndrome se constituya como una entidad nosológica, probablemente como consecuencia de la negativa experiencia de la terapia hormonal sustitutiva para el tratamiento de la menopausia (Rossouw, 2002). A este temor hay que sumar la debilidad del propio concepto del SDT: sus síntomas no sexuales son muy poco específicos y no está definido aún qué niveles de testosterona ni qué formas biológicas de la hormona son los más adecuados para llegar al diagnóstico. Asimismo, el reemplazo con testosterona conlleva riesgos, y los potenciales beneficios que se le atribuyen son escasamente relevantes (Gérvas, 2012). Sin embargo, desde 1990 las ventas de testosterona no han dejado de subir (Handelsman, 2012; Gérvas, 2012), lo que permite especular que es más producto de las estrategias de mercadotecnia que de la acumulación de evidencias científicas (Gorricho, 2012). En el tercer apartado del presente artículo analizaremos de qué manera los medios de comunicación, y en concreto la prensa escrita, han podido contribuir a este proceso.

\section{Los medios de comunicación como agentes medicalizadores}

La forma en que los medios de comunicación abordan la información en salud contribuye a acelerar los procesos por los que determinados problemas caen en el dominio de la medicina, ayudando así a la normalización del fenómeno de la medicalización (Pérez Oliva, 2009). En este apartado se desgranan algunos de los mecanismos por los que habitualmente los medios actúan como agentes medicalizadores, analizando el papel de los diversos agentes involucrados. 


\subsection{Los medios y su financiación}

Aunque en la Unión Europea no está permitida la publicidad directa de fármacos al consumidor, nada impide que haya informaciones en los medios donde se hable de dolencias concretas y de sus remedios. Es habitual que el paciente, tras tomar contacto con dichas noticias, acuda a su médico solicitando información sobre la enfermedad o una receta sobre dicho fármaco.

Una forma de promoción indirecta de enfermedades y medicamentos es a través de campañas de sensibilización, en cuya promoción participan habitualmente asociaciones profesionales y de pacientes, y que suelen estar financiadas por laboratorios farmacéuticos con interés en dar a conocer el trastorno (Ruíz-Cantero y CambroneroSaiz, 2011). No hay límites a la publicidad sobre estas campañas salvo el hecho de que no se mencionen marcas de fármacos $\mathrm{y}$, aunque las comunidades autónomas españolas tienen activados mecanismos de control, en numerosas ocasiones se consigue desvelar que esconden un fin comercial (Martín-Llaguno y Álvarez-Dardet, 2010). De hecho, en algunos casos la puesta en marcha de las campañas de sensibilización sobre enfermedades coinciden con el lanzamiento de un fármaco, y consiguen incrementar su demanda aunque no se mencionen directamente (Jong, 2004; Hall, 2011).

La publicidad de estas campañas, ya sea en anuncios o como publirreportajes, ha proporcionado durante muchos años una fuente de ingresos publicitarios importante para los periódicos. Pero no ha sido la única. Los laboratorios han elegido tradicionalmente los denominados "suplementos de salud" de los periódicos "generalistas" para divulgar "informaciones que aparecen en forma de noticias científicas pero que esconden un trasfondo de manipulación de los laboratorios farmacéuticos" (Elías, 2002). En España, gran parte de los grandes diarios de tirada nacional tenían su suplemento de salud. Ya que todos estos grandes medios forman parte de grupos empresariales con intereses diversos, los criterios periodísticos sobre la prioridad en los temas a tratar entran a veces en conflicto con dichos intereses (Gómez Martín, 2012; Pérez Oliva 2012). Esta influencia no determina sólo los contenidos, sino también la cantidad de veces que se trata en el medio un determinado tema. Dado que los medios de comunicación se realimentan, si la cobertura de un tema adquiere éxito lo habitual es que los medios de la competencia intenten también hacerse eco del mismo.

\subsection{Las fuentes y selección de la información}

Las fuentes de información de los periodistas sanitarios son varias. Cuando se trata de dar a conocer las innovaciones en el conocimiento de una enfermedad concreta, la más importante son las publicaciones en revistas biomédicas, que pueden llegar por varias vías: la institución que firma el estudio, la revista en sí y el laboratorio sobre cuyo fármaco trata el estudio. Esto último no es muy habitual, y solo suele ocurrir cuando el producto se considera 'revolucionario' (sucedió con la vacuna del papiloma). En los congresos médicos también de dan a conocer importantes avances científicos.

Otra fuente importante son las notas de prensa enviadas por los laboratorios, instituciones y agencias de comunicación (contratados por los primeros). Las notas de prensa 
pueden llegar directamente o distribuirse en una rueda de prensa, para la que se manda una convocatoria, algo que se suele reservar para noticias de mayor relevancia.

También existen los llamados "seminarios de formación", casi exclusivamente organizados por laboratorios farmacéuticos y con el apoyo de una asociación de pacientes o de profesionales (médicos, periodistas...). Estos seminarios suelen ser parciales y es el laboratorio el que elige (o al menos propone con mucho peso) docentes y temas. Las encuestas son otra fuente de noticias y hay que fijarse si son encuestas hechas con interés por parte del laboratorio. Por ejemplo, se puede hacer una encuesta con una buena muestra sobre el conocimiento de una determinada enfermedad, que demuestre que éste es ínfimo, de tal forma que se escriba sobre el tema y se contribuya así a su difusión y conocimiento, y con ello, voluntaria o involuntariamente, a su medicalización. Por último, la generalización de blogs y de nuevos perfiles de expertos informales ha abierto otra nueva vía de información a través de las redes sociales interactivas.

Sin embargo, no siempre las fuentes brindan información que pueda considerarse relevante o que suponga un avance real en la salud de las personas. Saber interpretar los hallazgos divulgados es imprescindible para ciudadanos, científicos, profesionales sanitarios y comunicadores (White, 2009). Los criterios que deben primar a la hora de que una noticia se refleje en los medios de comunicación deben ser que ésta aporte alguna novedad relevante y que suponga un problema de salud de interés general. Esto descarta a muchos seminarios de formación como fuentes de calidad, excepto cuando ofrezca la oportunidad de acceder a entrevistar a un experto que de otra manera sería inaccesible.

\subsection{Las agencias de comunicación}

Las agencias de comunicación son intermediarios entre sus clientes y los periodistas. En España existen varias agencias importantes especializadas en salud. Además de pasar las notas de prensa, es habitual que organicen encuentros amistosos entre periodistas del sector y representantes de los laboratorios, no para informar sobre nada concreto, sino para que se conozcan y el periodista pueda "poner cara" al laboratorio y viceversa. Son también las agencias las que se encargan de organizar viajes de prensa y de facilitar portavoces para distintos temas, por lo que su papel es importante para los periodistas.

\subsection{Conflictos de interés, transparencia e independencia profesional}

Existen varios puntos en los que se presentan potenciales conflictos de interés. El principal son los viajes de prensa (Pérez Oliva, 2010). Anteriormente, los periódicos, sobre todo los grandes, enviaban ellos mismos a sus periodistas a los congresos más relevantes. Ahora lo normal es que inviten laboratorios, puesto que los periódicos no tienen (o no quieren) emplear sus fondos para esos fines. Cuando se acude a un congreso invitado por un laboratorio existe el compromiso (no escrito, pero evidente) de 'cubrir' los eventos informativos que la empresa celebra; es decir, atender a la rueda de prensa. En general existe respeto al criterio profesional del periodista, pero quizás sea inevitable mirar con mayor simpatía las informaciones del que patrocina tu viaje. Ante 
esta situación, periódicos como El País incluyeron en su manual de estilo la obligación de detallar que el reportaje es fruto de un viaje pagado (1987), de igual manera que es habitual que en las publicaciones científicas sus autores declaren las fuentes de financiación de sus estudios y los potenciales conflictos de interés. Sin embargo, este ejercicio de transparencia puede ser interpretado por algunos como una forma de publicidad al obligar a mencionar el nombre del financiador.

Las farmacéuticas también organizan eventos directos de relaciones públicas. Son conocidas, por ejemplo, las cenas y algunos otros encuentros del sectori. Son actividades sociales, en las que se aprovecha para ver a los compañeros y hacer networking. También cuando la economía estaba mejor, solían enviar obsequios por navidad, normalmente respetando el valor máximo que tiene Farmaindustria (la patronal de la industria farmacéutica innovadora) en su código de buenas prácticas.

Otra práctica que genera potenciales conflictos de interés son los advisory boards. Son reuniones que organizan los laboratorios con periodistas muy selectos. En ellas el periodista aporta libremente a los responsables del laboratorio ideas sobre cómo mejorar la comunicación sobre un tema determinado, o bien traslada las cuestiones que más pueden interesar al ciudadano y al mundo de la comunicación sobre el tema de interés. La libertad de expresión es total, y no solo no se busca influir en el periodista para que escriba sobre nada determinado sino que incluso se firma un acuerdo de confidencialidad que impide precisamente que se divulguen sus contenidos. Son reuniones remuneradas (en torno a los 400 euros). Este tipo de reuniones no deberían comprometer la independencia profesional e incluso pueden contribuir a una comunicación más transparente, pero la realidad es que se cobra dinero de un laboratorio por una labor de asesoría.

Una actividad que es importante mencionar son los premios periodísticos. En su mayoría están organizados y patrocinados por laboratorios farmacéuticos, cuyo interés (que no esconden) es colocar en la agenda periodística un tema determinado. Son los premios periodísticos temáticos (sobre dolor, sobre osteoporosis, sobre cáncer de cabeza y cuello, por poner solo algunos ejemplos), aunque otras veces lo que se premia es la mejor información de salud en general o trayectorias profesionales. El conflicto de interés surge según el artículo se haya escrito con motivo del premio o no. En el primer caso, habría conflicto de interés y no tanto en el segundo. Aunque, de nuevo, es un asunto controvertido: ¿se tratará con la misma objetividad en el futuro al laboratorio que te ha premiado con 3.000 o 6.000 euros?

\subsection{Asociaciones de informadores y comunicadores en salud}

Las principales asociaciones de periodistas sanitarios en España son la Asociación Nacional de Informadores en Salud (ANIS) y la Asociación Española de Comunicación Científica (AECC), aunque no son las únicas. Algunas de ellas engloban a periodistas, pero también a fuentes. Esta convivencia, habitualmente provechosa, puede devenir en ocasiones en conflictos, ya que muchas veces las fuentes de noticias lo son también de ingresos. De hecho, existe una dependencia económica alta de algunas de estas asociaciones respecto a sus patrocinadores, algunas de ellas compañías farma- 
céuticas (PR Noticias, 2013). Por estos motivos, se hace exigible que dichas entidades se rijan por conductas y políticas de transparencia e independencia. Un papel primordial de estas asociaciones es el de promover y habilitar mecanismos que faciliten una mayor independencia profesional, sobre todo a través de la elaboración de códigos de conducta que regulen la forma en que financiadores y profesionales de la información se relacionan. Un buen ejemplo sería el de la Association of Health Care Journalists. En España aún no se ha profundizado mucho en este aspecto: la ANIS elaboró hace años su propio código, la Declaración de Sevilla, como un intento de regulación ética, aunque elude específicamente los temas más controvertidos (pago de viajes, etc.).

\section{La medicalización de la andropausia en la prensa}

La andropausia y la sexualidad en el varón maduro han sido temas recurrentes en la prensa española en los últimos 15 años, ya sea a partir de noticias sobre eventos o novedades, reportajes o entrevistas (tabla 1). Analizamos en este apartado cómo se ha desarrollado la cobertura mediática de estos temas (apartado 3.1) y cómo ésta ha servido para lanzar mensajes que han podido contribuir a su medicalización (apartado 3.2).

\subsection{Cobertura de la andropausia en medios de comunicación escritos}

Cuando se divulgan noticias sobre nuevos estudios, la información habitualmente procede de notas de prensa difundidas por las editoras de las revistas científicas. En ellas se suelen aportar datos concretos y citar las fuentes, permitiendo así que la información se pueda contrastar [referencias 4, 19, 29 y 21, tabla 1]. En ocasiones el redactor amplía la noticia con un reportaje que sitúa el problema en su contexto social e histórico, y donde se abre un debate en el que se tratan los puntos más controvertidos con información procedente de fuentes diferentes y a menudo contradictorias [2, 3, 11 y 18]. Añadir testimonios de pacientes es otro recurso habitual que añade credibilidad al texto, aunque a veces lo que se consigue con ello es justificar la medicalización [referencia 1, tabla 1]. En otros momentos son los propios promotores del estudio los que divulgan la noticia, adquiriendo habitualmente de esta forma un tono más promocional, más medicalizador y menos crítico [12].

Los congresos médicos son otra fuente de noticias sobre avances importantes [22]. Sin embargo, no siempre generan información original, y a veces incluso lo que trasciende de ellos son aspectos de escasa relevancia científica. Así, en la cobertura del $13^{\circ}$ Congreso Nacional de Andrología lo más destacado en prensa fue la mesa de clausura, moderada por un periodista y en la que participó una actriz y un perfumista [9]. En dicha mesa se dieron a conocer los resultados de la 'Il Encuesta Nacional sobre Salud del Hombre y la Andropenia' [10], encuesta cuyos resultados nunca han sido publicados en ninguna revista científica y que fueron obtenidos a partir de un cuestionario, el ADAM, que no está adaptado y validado para su uso en nuestro país, como se recomienda por organismos internacionales (Wild, 2005). Los resultados de dicha encuesta parecen desvelar una verdadera "epidemia" de andropausia, ya que "el $83.4 \%$ de los hombres presenta algún síntoma" del SDT. Si comparamos esta cifra con la de la primera edición de la encuesta (52\%) [5], pareciera como si en tan sólo 3 años se hubiera incrementado 
la prevalencia de este problema de una manera notable. Una lectura más detallada desvela que en la presentación de la segunda encuesta los datos aportados eran de la prevalencia de personas que habían respondido afirmativamente a cualquiera de los 10 ítems del cuestionario, cuando en realidad sólo podríamos considerar que su resultado es positivo si se responde afirmativamente a uno de los dos ítem de la esfera sexual o 3 del restante de las variables que mide el instrumento (Morley, 2000). Si además tenemos en cuenta de que dicho test no sirve como herramienta de cribado de la andropausia (Tancredi, 2004), que para el diagnóstico seguiría siendo necesario determinar los niveles hormonales (Morales, 2007) -cosa que no se hizo en dicha encuesta- y que la verdadera prevalencia del síndrome estaría más cercana a la cifra del $2 \%$ de la población de hombres de ese rango de edad (Wu, 2010), tenemos como resultado que el dato divulgado en dicho congreso es desproporcionado y contribuye a una alarma innecesaria.

Los foros de debate son otro foco, aunque escaso, de noticias. Su cobertura suele caracterizarse por su bajo perfil crítico: datos escasos, opiniones no contrastadas, falta de citación de las fuentes y omisión del origen de la financiación de los eventos [13]. Habitualmente son espacios en los que los expertos aprovechan para trasladar a la población masculina la necesidad de consultar al médico para diagnosticar y tratar la andropausia [14]. La alusión al infradiagnóstico y el desconocimiento sobre la enfermedad como lastres que impiden "avanzar en la calidad de vida general del hombre" es también usual [16]. Un ejemplo es el II Foro ABC-Salud, dedicado en esa edición a la salud del hombre y financiado por una compañía farmacéutica con intereses en el mercado de la testosterona y la disfunción eréctil. Una manera de cubrir eventos de este tipo es a partir del formato de entrevista al especialista participante. En este caso, fue un miembro del grupo de estudio de salud del hombre (GESH)ii, elaboradora también en las mencionadas encuestas. En la entrevista, el experto no duda en afirmar que "la sexualidad ha pasado de ser tratada sólo por psicólogos, que no son médicos, a ser parte de la medicina" [15], certificando así que se trata de un problema que ha caído en el dominio de los médicos [23].

Otra fuente de noticias son los seminarios de formación para medios de comunicación. Los impactos que surgen de estos encuentros suelen tener un contenido más pedagógico (origen del problema, síntomas asociados, formas de diagnóstico y alternativas terapéuticas), con lo cual son una buena manera de sensibilizar e informar a la población [6 y 7]. Dicho formato fue el elegido para divulgar precisamente los datos de la primera 'Encuesta Nacional sobre Salud del Hombre y la Andropenia'. El foro fue patrocinado por un fabricante de testosterona, que se encargó de lanzar las notas de prensa del evento, y contó con la participación de dos sociedades científicas [8].

Las campañas de sensibilización son, por último, un potente foco de noticias. Probablemente la más nombrada ha sido la campaña "Tenemos una edad" [17]. Además de servir para informar, en dicha campaña se han llevado a cabo actividades no respaldadas por la evidencia científica (Bhasin, 2010), como el ofreciendo gratuito de controles analíticos para determinar los niveles de testosterona ya sea en el lugar de trabajo (Limasa, 2011) o en los pasillos de un congreso científico (Sáinz, 2011), con el pretexto de ser una actividad encaminada a la salud laboral o para recabar muestras para un estudio científico, respectivamente. 
3.2 Contenidos y evolución histórica de los mensajes que han favorecido la medicalización de la andropausia

Aunque no han faltado reportajes que han hecho alusiones a la controversia en la consideración de la andropausia como una patología, el escepticismo sobre su diagnóstico y la desilusión sobre la falta de efectividad de la testosterona, en general, si atendemos a las frases recogidas en letra cursiva en el texto y que han sido seleccionadas de las fuentes que han servido como base para este artículo, los contenidos de las noticias han ido más en el sentido de promover la medicalización.

Muchos de los mensajes parecen querer despertar inicialmente una excesiva preocupación o incluso el miedo (al dotar a la andropausia de características temibles en cuanto a prevalencia y a pésimas repercusiones sobre la salud), para posteriormente ofrecernos una salida (ponerse en manos del experto) y una esperanza (en forma de suplementos de testosterona).

La testosterona se nos presenta como un remedio esencial para mantener la salud y prevenir la enfermedad (La testosterona es una hormona indispensable para la calidad de la vida, y puede prevenir muchas enfermedades. "Cuando se va la testosterona", ABC, 24 Mayo 2013), cuya duración en ocasiones debe ser para toda la vida (Experto citado en el artículo "El verdadero poder de la testosterona", El Mundo, 29 Noviembre 2010). A veces no se esconde que el objetivo último es prologar de forma artificial un estado lo más parecido a la juventud:

El tratamiento ideal sería aquel que "proporcionara un suministro de andrógenos que mantuviera en la sangre los niveles hormonales iguales a los del hombre joven". Experto citado en el artículo "Ellos también tienen 'menopausia", El País, 18 Diciembre 1995).

Se llega incluso a justificar el uso recreacional de los medicamentos en personas sanas que desean mejorar su desempeño sexual, lo cual es una forma de promover usos no autorizados de fármacos:

- [Periodista] Y no sólo para el que está malito: aconseja estos fármacos [para disfunciones sexuales] para mejorar al sano.

- [Experto] No tengo nada en contra del que quiera mejorar lo haga; pero si asume ese margen de mejora es que no está bien del todo. Por ejemplo, yo leo, pero si me pongo gafas leo mejor. Pues... ¿por qué no ponérmelas?

Experto citado en el artículo "Ignacio Moncada. El mal sexual ya no es de psicólogo: lo cura el médico, y con pastillas”, ABC, 4 Noviembre 2009.

Son numerosos los llamamientos a que el lector aludido consulte a un médico, generando un sentimiento de culpa por no "cuidarse" y no someterse de forma rutinaria a "controles". 
"El problema es que estos cambios [los debidos a la andropausia] se asocian a menudo con el paso del tiempo, la rutina... Los hombres se resisten y tardan más de un año, si finalmente dan el paso, en ir al médico. Es fundamental actuar ante estos síntomas". Experto citado en el artículo "Hombres sin testosterona", El Mundo, 22 de Junio 2010.

"Lo más difícil es que [los varones] vayan a las revisiones. Es algo consustancial a la condición masculina. Se asume que el hombre no tiene que estar enfermo. ¿A qué nos conduce? A que vivamos menos que las mujeres en todos los países". Experto citado en el artículo "Hay que hacer campañas de salud dirigidas a los hombres», ABC, 18 de Junio 2012.

De esta manera, se consigue llevar al lector a considerar que los cambios en el estado sexual del hombre maduro no son más que parte de una enfermedad cuya realidad es incuestionable, normalizando así la deriva medicalizadora de un proceso fisiológico.

La andropausia o 'pitopausia', como popular y jocosamente se la denomina a veces, no es un mito, sino una realidad, a pesar de que todavía hay algunos médicos y muchos hombres que la niegan. "Ellos también tienen 'menopausia'”, El País, 18 Diciembre 1995.

"Las medicinas han hecho que contemplemos las disfunciones sexuales como problemas médicos, que es lo que son". Experto citado en el artículo "Ignacio Moncada. El mal sexual ya no es de psicólogo: lo cura el médico, y con pastillas", ABC, 4 Noviembre 2009.

La evolución histórica de la distribución de las noticias en prensa sobre la andropausia sigue un patrón un tanto errático, como podemos ver en la tabla. Si bien cuando este síndrome fue relanzado a principios del siglo XXI algunos medios de comunicación se hicieron eco del renacimiento de la andropausia, tras la publicación del macroestudio Women's Health Initiative (Rossouw, 2002) y las dudas que arrojó sobre el enriquecimiento hormonal en la menopausia, las noticias sobre andropausia escaseaban. Todo cambió cuando los expertos, sus asociaciones profesionales y los laboratorios fabricantes de preparados de testosterona comenzaron a divulgar las encuestas y las campañas de sensibilización sobre el SDT, lo que ha originado varias oleadas de impactos en prensa en los últimos años y hasta nuestros días.

\section{Conclusiones}

Las fronteras de la medicina no cesan de ampliarse, incluyendo incluso procesos que podríamos considerar fisiológicos. El proceso normal de envejecimiento y sus consecuencias a nivel sexual, la andropausia, es un ejemplo paradigmático de este fenómeno. La reconsideración de este proceso como una entidad patológica ha resultado favorecido por los cambios recientes en el mercado farmacéutico de la sexualidad, y se ha acelerado como resultado de diversas estrategias de mercadotecnia. La disponibilidad de expertos y asociaciones profesionales a acrecentar su aceptabilidad social 
ha resultado también clave.

La influencia de los medios de comunicación sobre la opinión pública es indiscutible. Conocedores de esta cualidad, los actores interesados en sensibilizar sobre la andropausia han lanzado -mediante informaciones sobre encuentros con expertos, actividades científicas, encuestas, seminarios de formación y campañas específicas- numerosos mensajes que, en una importante proporción, han favorecido la medicalización de la sexualidad del varón maduro. Todo ello parece responder a una estrategia de comunicación muy definida y que en ocasiones parece entrar en conflicto con el rigor periodístico.

La independencia profesional del comunicador en salud y la transparencia en las relaciones entre fuentes, financiadores, informadores y grupos mediáticos son instrumentos imprescindibles para limitar la participación de los medios de comunicación como agentes que favorezcan la medicalización interesada o innecesaria. El papel de las asociaciones de profesionales de la información a este respecto puede ser fundamental. 
TABLAS

Tabla 1: Ejemplos de notas de prensa y de noticias aparecidas en España en medios escritos acerca de la andropausia y la sexualidad del varón maduro.

\begin{tabular}{|c|c|c|c|c|c|c|c|}
\hline \multirow[b]{2}{*}{$\mathbf{N}$} & \multirow[b]{2}{*}{ Referencia } & \multicolumn{6}{|c|}{ Contenidos } \\
\hline & & Reportaje & $\begin{array}{c}\text { Avance } \\
\text { investigación }\end{array}$ & $\begin{array}{c}\text { Congreso } \\
\text { o en- } \\
\text { cuentro } \\
\text { expertos }\end{array}$ & $\begin{array}{l}\text { Encuen- } \\
\text { tros con } \\
\text { periodistas }\end{array}$ & $\begin{array}{c}\text { Campañas } \\
\text { sensibilización }\end{array}$ & $\begin{array}{l}\text { Entre- } \\
\text { vista }\end{array}$ \\
\hline 1 & $\begin{array}{l}\text { Mayka Sánchez. } \\
\text { "Ellos también tie- } \\
\text { nen 'menopausia". } \\
\text { El País, 18-12- } \\
1995 .\end{array}$ & $X$ & & & & & \\
\hline 2 & $\begin{array}{l}\text { Lucía Argos. "La } \\
\text { testosterona que } \\
\text { viene". El País, 23- } \\
\text { 4-2000 }\end{array}$ & $X$ & & & & & \\
\hline 3 & $\begin{array}{l}\text { Gina Kolata. "La } \\
\text { terapia hormonal } \\
\text { masculina, bajo } \\
\text { sospecha". El País, } \\
\text { 10-9-2002 }\end{array}$ & $X$ & & & & & \\
\hline 4 & $\begin{array}{l}\text { Reuters. "Los altos } \\
\text { niveles de testoste- } \\
\text { rona provocan más } \\
\text { riesgo de cáncer de } \\
\text { próstata". El País, } \\
\text { 10-5-2004. }\end{array}$ & & $X$ & & & & \\
\hline 5 & $\begin{array}{l}\text { Nuria Ramírez de } \\
\text { Castro. "Más de la } \\
\text { mitad de los espa- } \\
\text { ñoles entre } 45 \text { y } 74 \\
\text { años tiene síntomas } \\
\text { de andropausia". } \\
\text { ABC, 13-11-2004. }\end{array}$ & & & & $X$ & & \\
\hline 6 & $\begin{array}{l}\text { Maite González. } \\
\text { "La crisis de los } \\
\text { ' } 45 \text { ' tiene solu- } \\
\text { ción". El Periódico } \\
\text { Mediterráneo, 21- } \\
11-2004 .\end{array}$ & & & & $X$ & & \\
\hline 7 & $\begin{array}{l}\text { "Andropenia, un } \\
\text { síndrome poco } \\
\text { conocido por los } \\
\text { hombres". El Día, } \\
\text { 1-12-2004. }\end{array}$ & & & & X & & \\
\hline
\end{tabular}




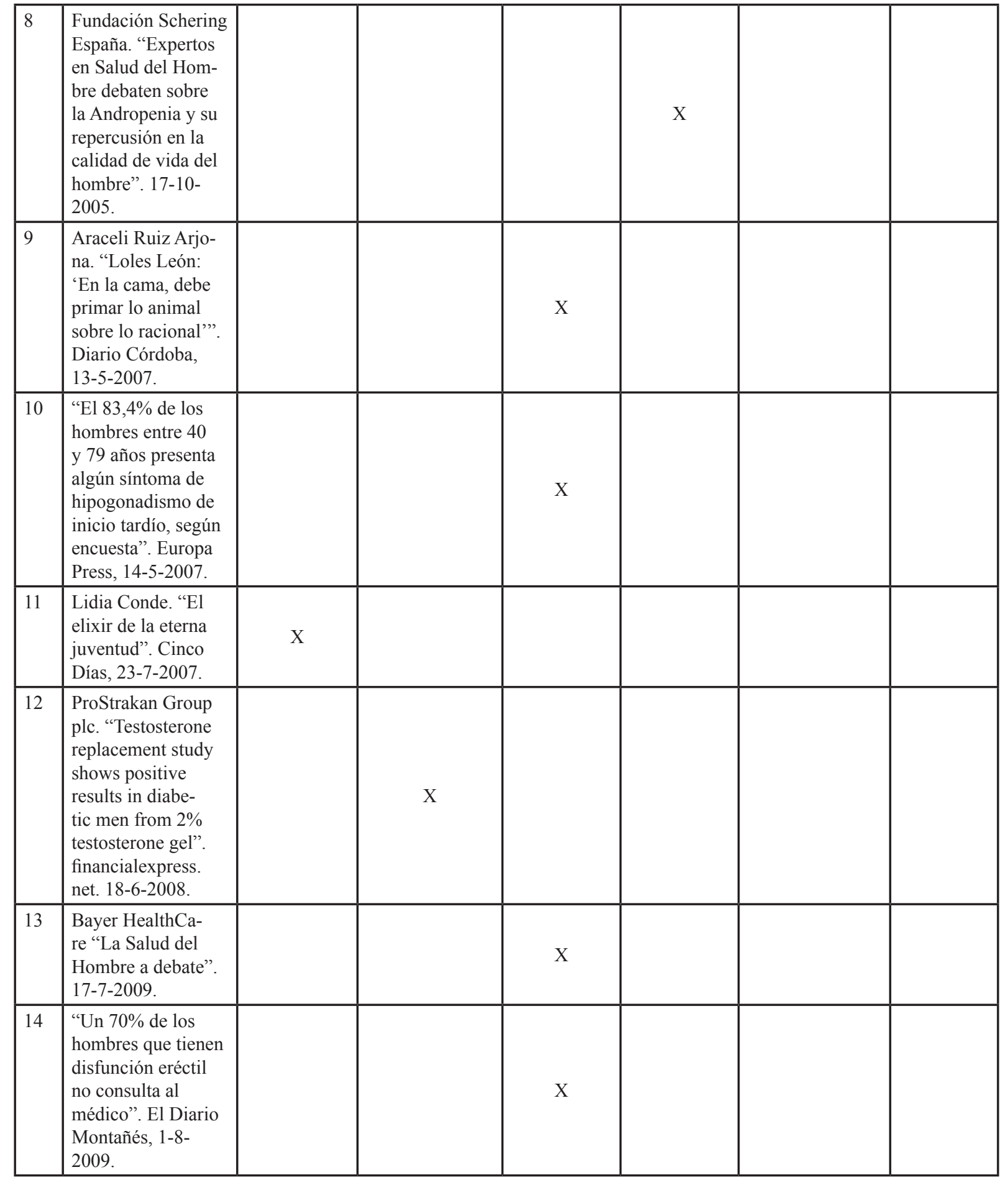




\begin{tabular}{|c|c|c|c|c|c|c|c|}
\hline 15 & $\begin{array}{l}\text { Ignacio Gil. "Ig- } \\
\text { nacio Moncada. El } \\
\text { mal sexual ya no } \\
\text { es de psicólogo: } \\
\text { lo cura el médico, } \\
\text { y con pastillas". } \\
\text { ABC, 4-11-2009. }\end{array}$ & & & & $X$ & & $X$ \\
\hline 16 & $\begin{array}{l}\text { "El } 30 \% \text { de los } \\
\text { casos de déficit de } \\
\text { testosterona no se } \\
\text { diagnostican bien". } \\
\text { Málaga Hoy, 18-3- } \\
2010 .\end{array}$ & & & $X$ & & & \\
\hline 17 & $\begin{array}{l}\text { Isabel L. Lantigua. } \\
\text { "Hombres sin } \\
\text { testosterona". El } \\
\text { Mundo, 22-6-2010. }\end{array}$ & & & & & $X$ & \\
\hline 18 & $\begin{array}{l}\text { María Sánchez- } \\
\text { Monge. "El } \\
\text { verdadero poder de } \\
\text { la testosterona". El } \\
\text { Mundo, 29-11- } \\
2010 . \\
\end{array}$ & $X$ & & & & & \\
\hline 19 & $\begin{array}{l}\text { Reuters. "El gel de } \\
\text { testosterona tiene } \\
\text { efectos en la diabe- } \\
\text { tes". 25-3-2011. }\end{array}$ & & $X$ & & & & \\
\hline 20 & $\begin{array}{l}\text { Frederik Joelving. } \\
\text { "Estudio cuestiona } \\
\text { relación entre tes- } \\
\text { tosterona y muerte } \\
\text { prematura". Públi- } \\
\text { co, 22-8-2011. }\end{array}$ & & $X$ & & & & \\
\hline 21 & $\begin{array}{l}\text { Frederik Joelving. } \\
\text { "Estudio liga enfer- } \\
\text { medad cardíaca a } \\
\text { bajos niveles de tes- } \\
\text { tosterona". Público, } \\
\text { 3-11-2011. }\end{array}$ & & $X$ & & & & \\
\hline 22 & $\begin{array}{l}\text { “¿Puede la terapia } \\
\text { de testosterona ayu- } \\
\text { dar a los hombres } \\
\text { obesos a perder } \\
\text { peso?” Medical } \\
\text { Express. 9-5-2012. }\end{array}$ & & $X$ & & & & \\
\hline 23 & $\begin{array}{l}\text { Pilar Quijada. «Hay } \\
\text { que hacer campañas } \\
\text { de salud dirigidas } \\
\text { a los hombres». } \\
\text { ABC, 18-6-2012. }\end{array}$ & & & & & $X$ & \\
\hline
\end{tabular}




\begin{tabular}{|l|l|l|l|l|l|l|l|}
\hline 24 & $\begin{array}{l}\text { Beatriz G. Portala- } \\
\text { tín. "Cuando se va } \\
\text { la testosterona". El } \\
\text { Mundo, 24-5-13. }\end{array}$ & & X & & & & \\
\hline
\end{tabular}

NOTA: Cada cita tiene su referencia en el texto entre corchetes.

\section{${ }^{i}$ NOTA}

7 El 23 de abril de 2013 se celebró en Madrid la 12a Velada del Periodismo Sanitario, con patrocinio de Novartis y la asistencia de numerosos periodistas y representantes de asociaciones de informadores en salud. Información obtenida de: http:// www.elglobal.net/elglobal/articulo.aspx?idart=747082\&idcat=784\&tipo=2

ii El llamado "Grupo de Estudio de Salud del Hombre", formado por 5 andrólogos y urólogos, es una marca registrada de la empresa Farmacéutica Bayer, que absorbió a Schering Plough, patrocinadora de las Encuestas Nacionales sobre Salud del Hombre y la Andropenia: http://www.informacion-empresas.com/Marca_GESHGRUPO-ESTUDIO-SALUD-HOMBRE.html

\section{Referencias}

Asociación Nacional de Informadores de la Salud (ANIS). Declaración de Sevilla. Principios para un ejercicio libre y responsable de la información en Salud. Disponible en: http://www.anisalud.com/sites/default/files/noticias/32283.doc. Recuperado el 12 de Junio de 2013.

Association of Health Care Journalists. Statement of Principles of the Association of Health Care Journalists. Disponible en: http://healthjournalism.org/secondarypage-details.php?id=56. Recuperado el 12 de Junio de 2013.

Ballard, Karen; Elston, Mary A. (2005). Medicalisation: A multi-dimensional concept. Social Theory \& Health. Vol. 3, n³, pp. 228-241.

Barsky, Arthur J.; Borus, Jonathan F. (1995). Somatization and medicalization in the era of managed care. Journal of the American. Medical Association. Vol 274, $\mathrm{n}^{\circ} 24$, pp. 1931-1934.

Bhasin, S. ; Cunningham, G. R. ; Hayes, F. J. ; Matsumoto, A. M. ; Snyder, P. J. y Swerdloff, R. S. (2010). Testosterone therapy in men with androgen deficiency syndromes: an Endocrine Society clinical practice guideline. Journal of Clinical Endocrinology and Metabolism. Vol. 95, pp. 2536-2559.

Broom, Dorothy H. y Woodward, Roslyn V. (1996). Medicalisation reconsidered: toward a collaborative approach to care. Sociology of Health \& IIIness. Vol. 18, n 3 , pp. 357378. 
Brown-Séquard, Charles. (1889). Effects produced on man by subcutaneous injections of a liquid obtained from the testicles of animals. The Lancet. Vol. 2, pp. 105-107.

Conrad, Peter. (2007). The Medicalization of Society: On the Transformation of Human Conditions into Treatable Disorders. Baltimore: Johns Hopkins University Press.

Conrad, Peter. (1992). Medicalization and social control. Annual Review of Sociology. Vol. 18; pp. 209-232.

Conrad, Peter, y Leiter, Valerie. (2004). Medicalization, markets and consumers. Journal of Health and Social Behavior. Vol 45 (Extra Issue), pp. 158-176.

Conrad, Peter; Mackie Thomas y Mehrotra, Ateev. (2010). Estimating the costs of medicalization. Social Science \& Medicine. Vol. 70, nº 12, pp. 1943-1947.

El País. (1987) Manual de estilo. Disponible en: http://blogs.elpais.com/files/manualde-estilo-de-el-pa\%C3\%ADs.pdf. Recuperado el 12 de Junio de 2013.

Elías, Carlos. (2002). Los suplementos especializados como guetos de noticias en la prensa generalista: el caso de los científicos y sanitarios. Ámbitos. № 9-10, pp. 171186. Disponible en: http://grupo.us.es/grehcco/ambitos09-10/elias.pdf. Recuperado el 12 de Julio de 2013.

Gérvas, Juan; Gavilán, Enrique y Gorricho, Javier. (2012). Medicalización del envejecimiento: síndrome por déficit de testosterona. Boletín de Información Terapéutica de Navarra. Vol. 20, $\mathrm{n}^{\circ} 4$, pp. 1-8. Disponible en http://www.navarra.es/NR/ rdonlyres/0220315B-65F6-44DA-A97A-7B1810DD2B39/237520/Bit_v20n5.pdf. Recuperado el 12 de Junio de 2013.

Gómez Martín, Paz. ¿Crisis sanitarias o crisis mediáticas? En: de Semir, Vladimir y Revuelta, Gema. (2012). El periodismo biomédico en la era 2.0. Cuadernos de la Fundación Dr. Esteve, nº 25. Barcelona: Fundación Dr. Antonio Esteve.

Gorricho, Javier; Gavilán, Enrique y Gérvas, Juan. (2012). Marketing, not evidence based arguments, has probably increased testosterone prescribing. British Medical Journal. Vol. 345, pp. e6905.

Greenhalgh, Trisha y Wessely, Simon. (2004). 'Health for me': a sociocultural analysis of healthism in the middle classes. British Medical Bulletin. Vol. 69, n 1, pp. 197-213.

Groopman, Jerome. (2002) Hormones for men. Is male menopause a question of medicine or of marketing? New Yorker. Disponible en: http://www.newyorker.com/ archive/2002/07/29/020729fa_fact. Recuperado el 12 de Junio de 2013.

Hall, Danika; Jones, Sandra e Iverson, Don. (2011). Disease awareness advertising: women's intentions following exposure. Australian Family Physician. Vol. 40, n 3, pp. 143-147. 
Handelsman, David J. (2012) Pharmacoepidemiology of testosterone prescribing in Australia, 1992-2010. Medical Journal of Australia. Vol. 196, n 10, pp. 642-645.

Illich, Ivan. (1975). Némesis médica: la expropiación de la salud. Barcelona: Barral Editores.

Jong, Geert W.; Stricker, Bruno H. y Sturkenboom, Miriam C. (2004). Marketing in the lay media and prescriptions of terbinafine in primary care: Dutch cohort study. British Medical Journal. Vol. 328, nº 7445, p. 931.

Khan, Arnold, (2005). Regaining lost youth: the controversial and colourful beginnings of hormone replacement therapy in aging. The Journals of Gerontology: Series A: Biological Sciences and Medical Sciences. Vol. 60, n² 2, pp. 142-147.

Limasa, Servicios de Limpieza Integral de Málaga III, SA. (2011). Boletín informativo Septiembre, $n^{\circ}$ 1. Disponible en: http://www.limasa3.es/sites/default/files/Boletin\%20Informativo\%20Limasa\%20-\%20Septiembre\%202011.pdf. Recuperado el 12 de Junio de 2013.

Marañón, Gregorio. (1937). Climaterio de la mujer y del hombre. Madrid: Espasa-Calpe.

Martín-Llaguno, Marta y Álvarez-Dardet, Carlos. (2010). La retirada de una campaña publicitaria para promoción de la vacuna tetravalente del virus del papiloma humano en España. Gaceta Sanitaria. Vol. 24, n¹, pp. 75-77.

Morales, A.; Spevack, M.; Emerson, L.; Kuzmarov, I.; Casey, R.; Black, A. y Tremblay, R. (2007). Adding to the controversy: pitfalls in the diagnosis of testosterone deficiency syndromes with questionnaires and biochemistry. Aging Male. Vol. 10, n² 2, pp. 57-65.

Morley, J. E.; Charlton, E.; Patrick, P.; Kaiser, F. E.; Cadeau, P.; McCready, D. y Perry, H. M. (2000). Validation of a screening questionnaire for androgen deficiency in aging males. Metabolism. Vol 49, n 9, pp. 1239-1242.

Null, Garry; Feldman, Martin; Rasio, Debora y Dean, Carolyn. (2010). Death by medicine. Mount Jackson: Praktikos Books.

Parens Erik. (2013). On good and bad forms of medicalization. Bioethics. Vol. 27, $\mathrm{n}^{\circ} 1$, pp. 28-35.

Pérez Oliva, Milagros. (2009). Expectativas de salud en la sociedad mediática global. Humanidades Médicas. Diciembre, n45.

Pérez Oliva, Milagros. (2010). Avances médicos con intereses ocultos. El País. 17 Enero.

Pérez Oliva, Milagros. Periodismo de calidad en tiempos de Internet. En: de Semir, Vladimir y Revuelta, Gema. (2012). El periodismo biomédico en la era 2.0. Cuadernos de la Fundación Dr. Esteve, n² 25. Barcelona: Fundación Dr. Antonio Esteve. 
PRNoticias, Redacción. (2103). Asamblea General de ANIS: El G20 sanitario arremete contra la gestión de la anterior junta. PRNoticias, 17 Junio. Disponible en: http://www. prnoticias.com/index.php/salud/762/20122481. Recuperado el 12 de Julio de 2013.

Rossouw, J. E.; Anderson, G. L.; Prentice, R. L.; LaCroix, A. Z.; Kooperberg, C.; Stefanick, M. L., et al. (2002). Risks and benefits of estrogen plus progestin in healthy postmenopausal women: principal results From the Women's Health Initiative randomized controlled trial. Journal of American Medical Association. Vol. 288, nº 3, pp. 321333.

Ruíz-Cantero, María T. y Cambronero-Saiz, Belén. (2011). La metamorfosis de la salud: invención de enfermedades y estrategias de comunicación. Gaceta Sanitaria. Vol. 25, n० 3, pp. 179-181.

Sáinz Corada, E. (2011). Pros y contras del abordaje del SDT. Gaceta Médica. Disponible en http://www.gacetamedica.com/gaceta/articulo.aspx?idart=534155\&idcat=703 \&tipo=2

Tancredi, A.; Reginster, J. Y.; Schleich, F.; Pire, G.; Maassen, P.; Luyckx, F. y Legros, J. J. (2004). Interest of the Androgen Deficiency in Aging Males (ADAM) questionnaire for the identification of hypogonadism in elderly community-dwelling male volunteers. European Journal of Endocrinology. Vol. 151, n 3, pp. 355-360.

Watkins, Elizabeth S. (2008). Medicine, masculinity, and the disappearance of male menopause in the 1950s. Social History of Medicine. Vol. 21, n² 2, pp. 329-344.

Watt, Graham. (2002). The inverse care law today. The Lancet. Vol. 360, pp. 252-254.

White, Alicia. (2009). How to read health news. Disponible en: http://www.nhs.uk/news/ Pages/Howtoreadarticlesabouthealthandhealthcare.aspx. Recuperado el 12 de Junio de 2013.

Wild, D.; Grove, A.; Martin, M.; Eremenco, S.; McElroy, S.; Verjee-Lorenz, A. y Erikson, P. (2005). Principles of Good Practice for the Translation and Cultural Adaptation Process for Patient-Reported Outcomes (PRO) Measures: Report of the ISPOR Task Force for Translation and Cultural Adaptation. Value in Health. Vol. 8, n² 2, pp. 94-104.

Wu, F. C.; Tajar, A.; Beynon, J. M.; Pye, S. R.; Silman, A. J.; Finn, J. D.; et al. (2010). Identification of late-onset hypogonadism in middle-aged and elderly men. New England Journal of Medicine. Vol. 363, n² 2, pp. 123-135. 